\title{
Phase Separation Phenomena During the Formation of Asymmetric Membranes
}

\author{
D. M. KOENHEN, M. H. V. MULDER,* and C. A. SMOLDERS, Twente \\ University of Technology, Enschede, The Netherlands
}

\begin{abstract}
Synopsis
The formation of membranes from two systems has been studied. In the system polyurethanedimethylformamide-water, the mechanism for the formation of the sponge-like structure proves to be a liquid-liquid phase separation with nucleation and growth of the diluted phase. This mechanism has been confirmed for the system modified polystyrene-polyisoprene-polystyrene/ $o$-dichlorobenzene/(methanol-water). Crystallization and gelation is discussed. The membranes prepared showed hyperfiltration activity. The mechanism proposed here is believed to be valid for other systems, too.
\end{abstract}

\section{INTRODUCTION}

Loeb and Sourirajan ${ }^{1}$ were the first to develop a method for preparing asymmetric ("high flux") membranes for the application of desalination by reverse osmosis. These membranes consist of an extremely thin selective layer (0.1-0.2 $\mu \mathrm{m})$ backed by a porous substructure in which sometimes finger-like cavities can be present. In principle, these asymmetric membranes were obtained by the following procedure: After casting a solution of cellulose acetate (CA) on a glass plate, the volatile solvent (acetone) was allowed to evaporate for a short time, followed by immersion in a precipitating agent (for instance, water). Heat treatment of the final membrane will improve the salt rejection.

Many investigations have been dedicated to elucidate the formation mechanisms of the asymmetric structure of the membranes. These investigations cover roughly the following approaches: (1) An explanation is given of the phase transition, or phase inversion, during the formation of the membrane structure. (2) An explanation for the final asymmetric structure is brought in relation to physical quantities of the materials used and the conditions in which the membranes are prepared.

In the first group of investigations, several authors explained the formation of the sponge-like structure in a way very similar to the colloidal demixing theory described by Bungenberg de Jong. ${ }^{2}$ To this group of authors belong Maier and Scheuermann, ${ }^{3}$ Keilin, ${ }^{4}$ and Kesting. ${ }^{5}$ In the mechanism described, spherical droplets or micelles are formed in which the mass of the polymer molecules is concentrating at the exterior surfaces. Subsequently, the micelles will contact each other and form polyhedra. The process is completed by further polymer

* Present address: Wafilin B. V., Hardenberg, The Netherlands. 
desolvation, syneresis, and capillary depletion. Strathmann, Scheible, and Baker, ${ }^{6}$ making use of ternary phase diagrams, described how the polymer-rich phase separates from a supersaturated solution to form spherical solid droplets, which can grow and agglomerate to form an interconnected solid structure. In a later report, Strathmann et al. ${ }^{7}$ revised this mechanism in such a way that the precipitated polymer-rich phase remains fluid for some time and agglomerates to form finally a solid polymer matrix.

In the second group of investigations, Frommer and Lancet ${ }^{8}$ and Strathmann et al. ${ }^{7}$ have related the final asymmetric structure to the rate of precipitation. When the rate of precipitation is low, a sponge-like structure will be obtained. These authors also studied the formation of finger-like cavities which are often found in asymmetric membranes. They concluded that the formation of finger-like cavities, growing in the fluid layer, is generally associated with a high rate of precipitation.

Matz ${ }^{9}$ explained the formation of finger-like cavities on the basis of changing surface tension during the coagulation step. The surface tension of the polymer-rich phase increases through loss of the solvent, while the surface tension of the aqueous phase will decrease. At the point where these surface tensions are equal, the interface is very sensitive to distortion and cavity formation is easily initiated. Growth can occur through diffusion of the solvent into these cavities.

Another explanation was proposed by Strathmann et al. ${ }^{7}$ who stated that the homogeneous skin layer cracks through shrinkage of the polymer-rich phase and cavities can develop. Growth of cavities proceeds by shrinkage of the polymer matrix which is caused by inflow of nonsolvent.

Much the same type of coagulation process which occurs during the formation of asymmetric membranes is encountered during the wet-spinning process of synthetic polymer fibers. The presence of finger-like cavities in the fibers has been reported, for instance, for wet-spun acrylic fibers ${ }^{10,11}$ and for nylon 6 and polyurethane. ${ }^{12}$

According to Gröbe et al. ${ }^{11}$ the mass transfer will be favored in places of the polymer-poor phase. The number of these places is given by statistical distribution. The phase separation front will move faster in these places because of the faster exchange of solvent and nonsolvent. Because there is nonsolvent present in the polymer-poor dilute phase, solvent can reduce its chemcal potential by diffusing into it.

Eventually, the cavity will contain so much of the solvent that the composition is below the precipitation threshold. Coagulation at the boundary of the cavity will then be retarded. Solvation of the polymer molecules in the solution, just outside the cavity, is lowered by diffusion of the solvent into the cavity. Therefore, transfer of the polymer will occur to surrounding places by creep; eventually, complete desolvation will take place.

In this paper, we will report on experiments done in our laboratory to obtain more information concerning the phase separation involved in the membrane formation. Originally, this work was started to provide insight in the coagulation process as a part of a program on wet-spun fibers. Later, it appeared to us that it would also be applicable to the processes occurring during the formation of asymmetric membranes. The demixing process will be discussed taking the line of the thermodynamics and kinetics of phase equilibria in polymer solutions. 


\section{THEORETICAL CONSIDERATIONS}

\section{Liquid-Liquid Phase Separation}

The Gibbs free enthalpy of mixing of a polymer and a solvent can be described with the Flory-Huggins equation ${ }^{13}$ which is expressed here in weight fractions:

$$
\frac{\Delta G_{m}}{R T}=w_{0} \ln w_{0}+\sum_{i} \frac{M_{0}}{M_{i}} w_{i} \ln w_{i}+g \Sigma w_{i} \cdot w_{0}
$$

in which $\Delta G_{m}=$ free enthalpy of mixing, $R=$ universal gas constant, $T=$ absolute temperature, $w_{\mathrm{o}}=$ weight fraction of solvent, $w_{i}=$ weight fraction of polymer species $i, M_{o}=$ molecular weight of the solvent, $M_{i}=$ molecular weight of the polymer species $i$, and $g=$ empirical correction factor. The first two terms on the right-hand side of eq. (1) give the entropy of mixing. This entropy is small compared with the mixing of low molecular weight substances through the factor

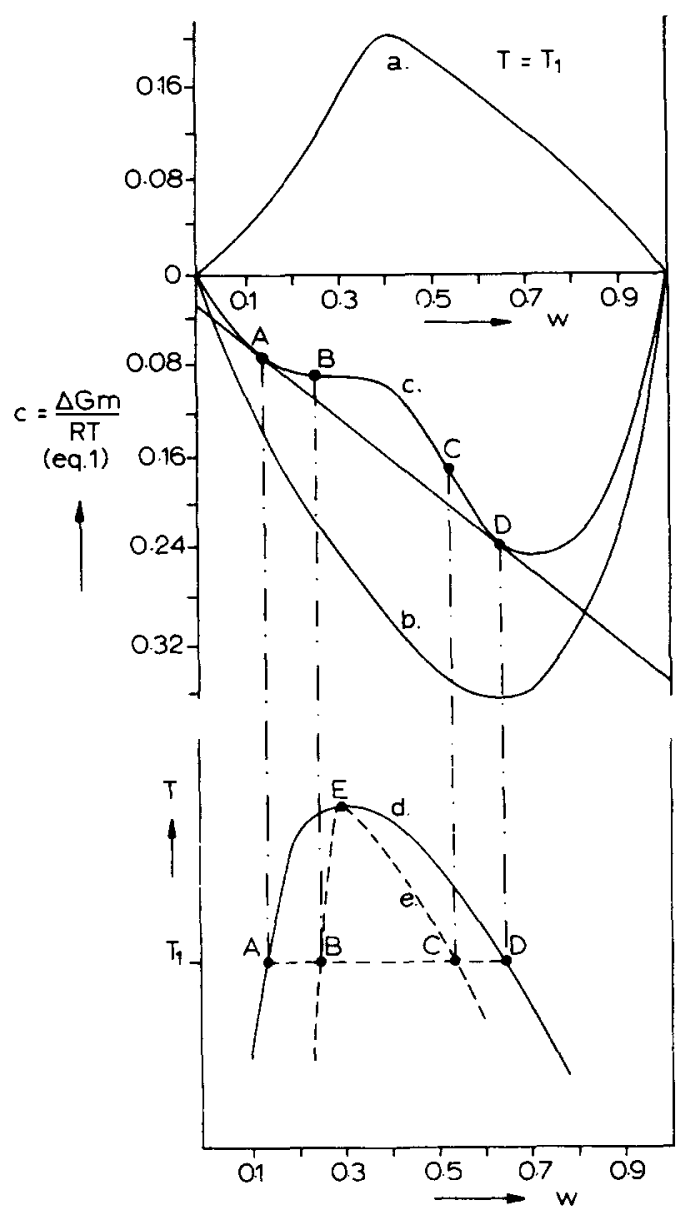

Fig. 1. Schematic representation of the free enthalpy components given by eq. (1) and the corresponding phase diagram for a binary system with liquid-liquid phase separation: curve a, $g \Sigma w_{i} w_{0}$; curve b; $w_{o} \ln w_{o}+\Sigma_{i}\left(M_{o} / M_{i}\right) w_{i} \ln w_{i} ;$ curve $c, \Delta G m / R T$; curve d, cloud point curve, curve e, spinodal. 


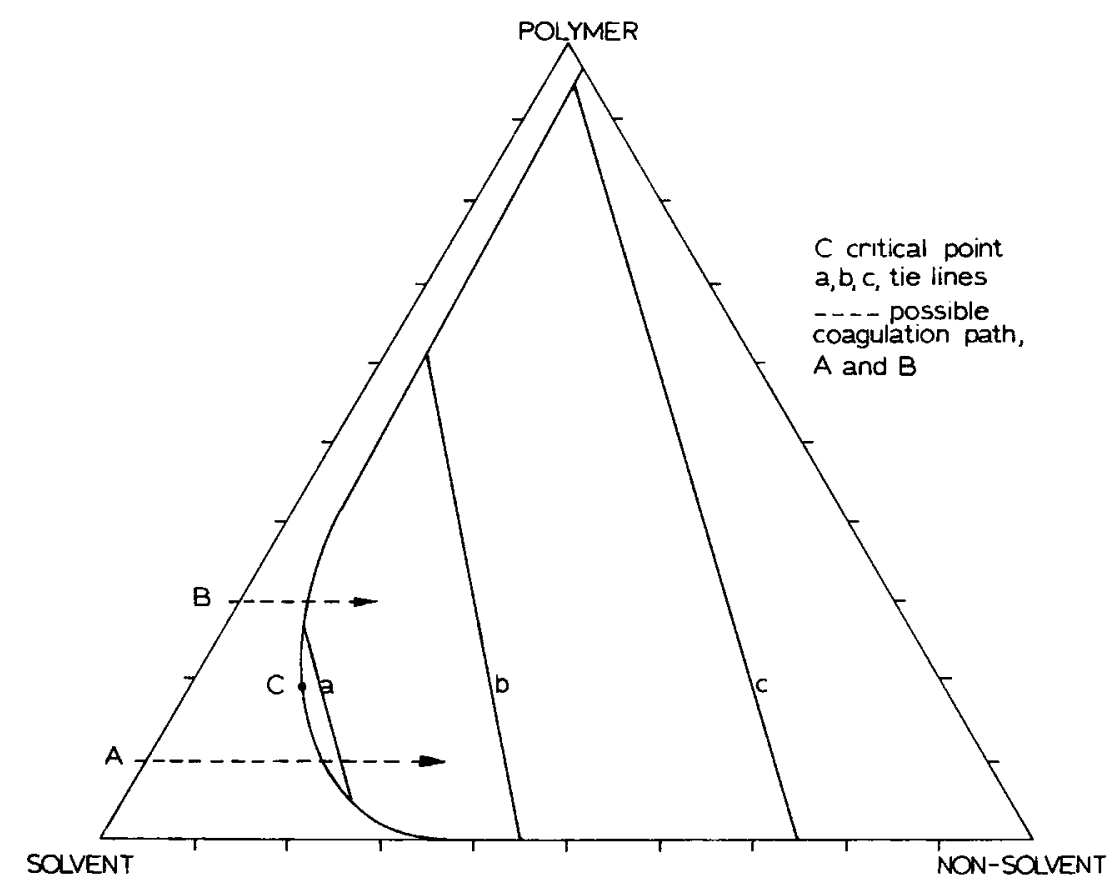

Fig. 2. Schematic phase diagram for a ternary system.

$M_{o} / M_{i}$. Because this entropy of mixing is small, a small endothermic enthalpy of mixing or some other nonideality, together represented by the positive term $g \sum w_{o} w_{i}$, can cause a phase separation.

The situation in such a case is given schematically in Figure 1, where points $\mathrm{A}$ and $\mathrm{D}$ are the common tangents of the $\Delta G_{m}$ curve. These concentrations $\mathrm{A}$ and $\mathrm{D}$ have identical chemical potentials and form phases in equilibrium. The points $\mathrm{B}$ and $\mathrm{C}$ are the points of inflection of the $G_{m}$ curve. Any composition between points $A$ and $D$ can lower its free enthalpy of mixing by separating into phases of compositions A and D. Lines connecting the compositions of the coexisting phases (A-D, etc.) are called tie lines. At point $\mathrm{E}$, the compositions are the same and the tie line is a point, the critical point. Compositions between $A$ and $B$ on one side of the demixing region and $C$ and $D$ on the other side are metastable and only give demixing when a nucleus is formed having a composition which is close enough to the composition at the other end of the demixing gap. These nuclei grow further until a complete demixing into phases $\mathrm{A}$ and $\mathrm{D}$ has taken place. This mechanism is called nucleation and growth.

The compositions between the points of inflection $\mathrm{B}$ and $\mathrm{C}$ are unstable to the very small fluctuations which are always present. Such compositions would give instantaneous demixing, the so-called spinodal demixing. This type of demixing, however, is difficult to obtain in fluid systems because in most cases nucleation and growth will occur in passing the metastable region.

In a system which is composed of a polymer and one or more solvents and nonsolvents, which is the case in membrane preparation, there are more degrees of freedom. The phase diagram of a ternary system consisting of a polymer, a 
solvent, and a nonsolvent is most conveniently given by the so-called isothermal sections (Fig. 2). When a polymer solution is coagulated with a nonsolvent as in membrane preparation, we can assume that after the initial skin formation, the overall polymer concentration in the membrane is constant, while the concentration of solvent decreases and that of nonsolvent increases.

In a system as illustrated in Figure 2, a solution of, say, $10 \%$ polymer passes the border of the two-phase region at the diluted side of the critical point, whereas a solution of $30 \%$ enters this region at the opposite side of the critical point. In the first case, nuclei of the concentrated phase will be formed with a large difference in composition from the original solution, and the diluted phase will change continuously in composition, getting more and more dilute. In the latter case, nuclei of the diluted phase will be formed while the concentrated phase can change continuously. Thus, in nucleation and growth, we have two possibilities: (A) nucleation and growth of the concentrated phase, and (B) nucleation and growth of the diluted phase

The consequences for the morphology in the final coagulated polymer solution are obvious. In case $A$, one expects spheres of the concentrated phase dispersed in a dilute solution. In case $B$, one expects spheres of a dilute solution surrounded by concentrated polymer solution matrix. When the precipitation process has come to an end, the concentration of polymer in the concentrated phase has become very high, i.e., the polymer has solidified and the following morphologies can exist: In case A, a latex is obtained which in principle has no mechanical strength. Through coalescence or sticking of the polymer spheres, an open-pore structure can be obtained which could have some strength. In case $\mathrm{B}$, spherical cells will be formed.

\section{Crystallization}

Crystallization is another type of phase separation which is possible in solutions of macromolecules. From dilute solutions, some polymers can crystallize in the form of lamellar single crystals being about $100 \AA$ thick and often many microns in the lateral directions. In these crystals, the polymer chains are oriented normal to be plane of the lamellea and are arranged in a folded manner. ${ }^{16}$ From concentrated solutions, more complicated crystal structures can be obtained, reminiscent of the familiar spherulites obtained by crystallization from the melt. The melting points of these crystallites can be described qualitatively by the Flory melting point depression theory. ${ }^{13}$

When the concentration is high or when the crystallites are small, many links between different polymer molecules are formed, and a homogeneous but cloudy gel can be obtained. These gels are thermoreversible, and the melting points can also be described by the Flory therory. ${ }^{17}$ The gel structure is thought to be composed of the familiar fringed micelles. Often, the formation of helices is involved in the crystallization and gelling process. ${ }^{18,19,20}$ Gelation can also occur in solutions of the well-known membrane material cellulose acetate. ${ }^{21}$ These considerations will be of importance for the solidification (gelation) of concentrated polymer regions in membranes, like the "skin" at the surface of the membrane, or the concentrated polymer phase which forms the matrix in the porous part of the membrane. 


\section{EXPERIMENTAL}

\section{Materials}

Two different types of membranes have been prepared: (a) from polyurethane (PU) (Estane $5701 \mathrm{~F} 1$ ), and (b) from a modified polystyrene-polyisoprenepolystyrene (SIS) block copolymer (CARIFLEX TR 1108). All of the solvents used were of analytical grade.

\section{Cloud Points}

Cloud points were determined in well-homogenized PU solutions. Homogeneous mixtures of polyurethane-dimethylformamide were prepared by the method also used by Van Emmerik and Smolders. ${ }^{22}$

The appropriate amounts were weighed in small Pyrex glass tubes which were degassed, flushed with nitrogen, degassed again, and sealed under vacuum at liquid nitrogen temperature. The sealed glass tubes were heated, if necessary, in an oil bath to approximately $80^{\circ} \mathrm{C}$ for at least $24 \mathrm{hr}$ in order to obtain homogeneous liquid mixtures.

Phase separation points were usually determined by cooling or heating the thermostat bath $1^{\circ} \mathrm{C}$ every hour or $1^{\circ} \mathrm{C}$ every 10 min.

\section{Optical Microscope Studies}

The phase separation of polyurethane was examined with a Olympus EH microsope. A drop of polymer solution is placed between two microscope slides. By means of a hypodermic syringe, water is placed near the edge. Photographs are taken of the interface at certain time intervals.

\section{Membrane Preparation}

PU Membranes. PU membranes were prepared from 15-30\% polyurethane solutions in DMF. The solution was cast on a glass plate and, after a short evaporation time, immersed in a coagulation bath. Water and different ratios of DMF/water solutions were used as precipitation media.

Ionic Membranes. These were prepared from polystyrene-polyisoprenepolystyrene (SIS) block copolymer which was modified as follows. ${ }^{23}$ To a $10 \%$ SIS solution in toluene, N-chlorosulfonyl isocyanate (NCSI) was added at room temperature. Below, the reaction of the polyisoprene middle block with NCSI is shown schematically:

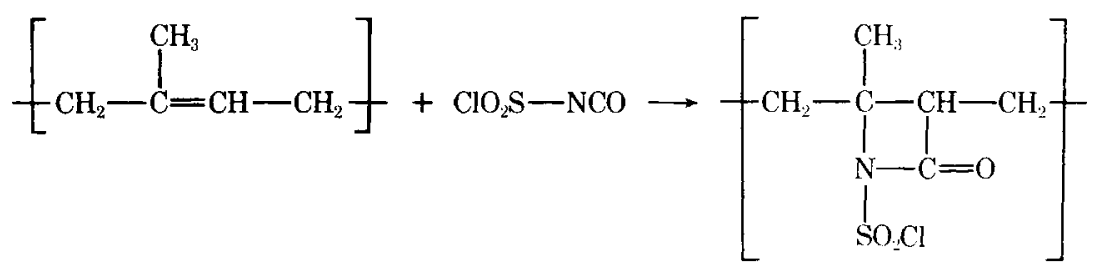


The molar ratio of NCSI added to isoprene was 60\%. After isolation of the product, the polymer was dissolved in 1,2-dichlorobenzene.

A $15 \%$ solution of compound I in 1,2-dichlorobenzene was cast on a glass plate. After a certain evaporation time, the cast polymer was immersed into a water/ methanol (20/80) bath. In order to prevent extensive swelling, crosslinks were introduced by immersing the membrane in a $2 \%$ hexamethylenediamine solution in toluene at room temperature. In a final step, the membrane was modified by immersing it at room temperature in a concentrated $(4 M)$ ammonia solution in which hydrolysis occurs, giving polymer II:

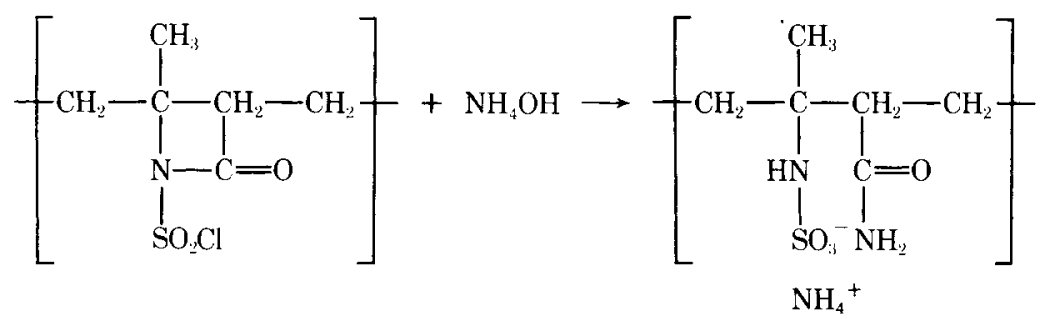

(II)

\section{Scanning Electron Microscope Studies}

Cross sections of the membranes were examined with a JEOL-JSM U3 scanning electron miscroscope (SEM). Samples were prepared by cryogenic breaking followed by freeze drying (or etching) and coating the sample with a chargeconducting layer according to a technique developed earlier. ${ }^{12}$

\section{Hyperfiltration Experiments}

Hyperfiltration experiments were carried out in a stirred Amicon high-pressure cell, Type 420 , at an operating pressure of 40 atmospheres. In each experiment, a $3000 \mathrm{ppm} \mathrm{NaCl}$ solution was used as feed solution. The concentrations in feed and product were determined by specific resistance measurements using a conductivity cell.

\section{RESULTS}

In the quasi-ternary system of polyurethane-dimethylformamide-water, quasi-binary sections were chosen for which the following phase transitions were studied.

\section{Liquid-Liquid Phase Separation}

In the first place, a range of polymer solutions with a constant ratio of dimethylformamide-water were studied. When a homogeneous solution is cooled, cloudiness appears at a certain temperature. The appearance of the cloudiness is not dependent on the rate of cooling; and when the solution is centrifuged, two liquid layers can be obtained in the tube. By heating the phase-separated solution, the system becomes homogeneous again at the same temperature. Hence, a liquid-liquid phase separation is obtained in these experiments. The cloud 


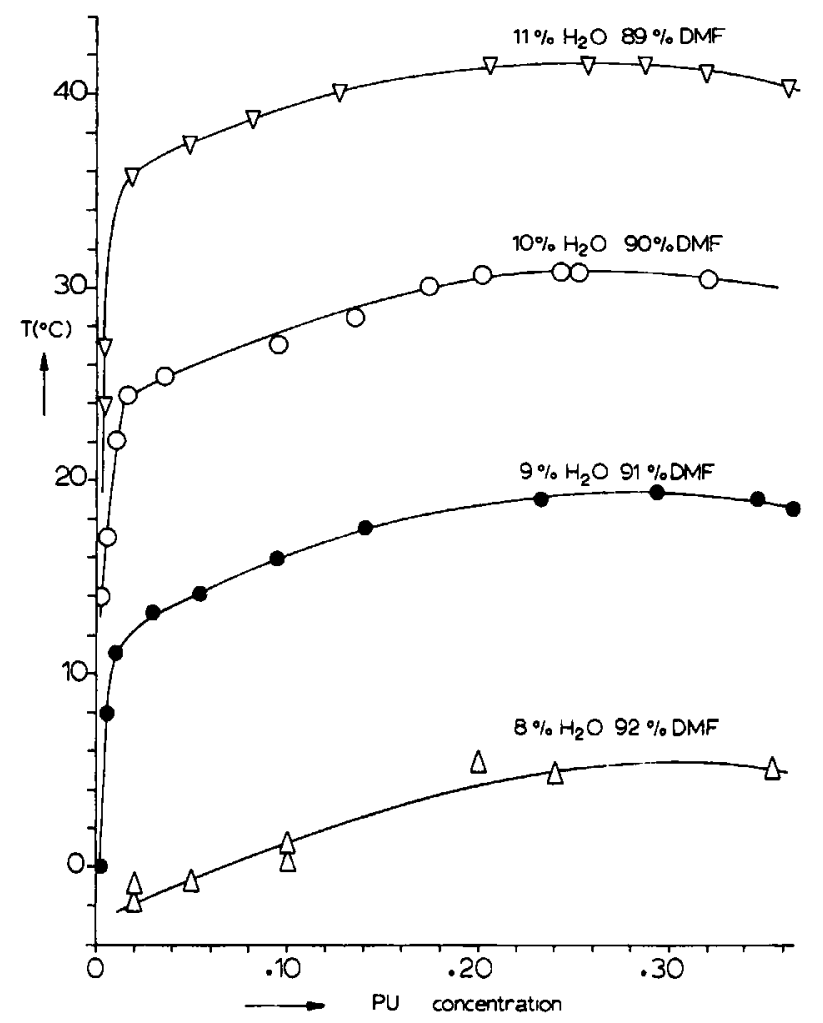

Fig. 3. Cloud point curves in the system PU/DMF/ $\mathrm{H}_{2} \mathrm{O}$.

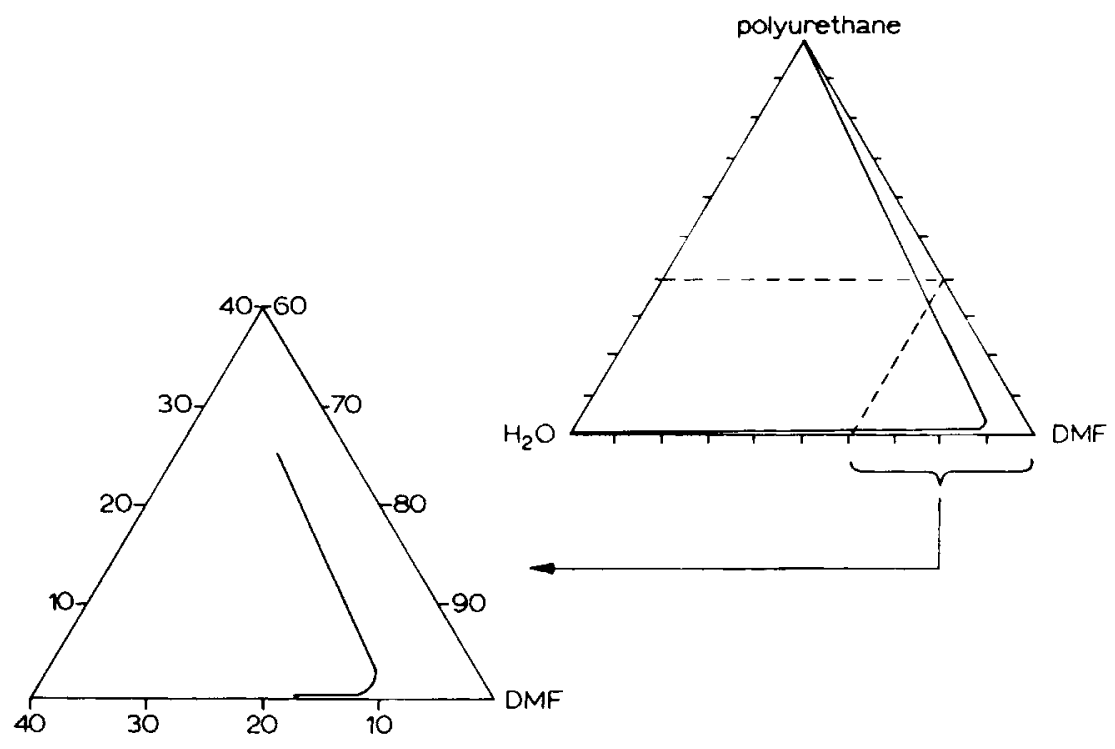

Fig. 4. Ternary diagram for $\mathrm{PU} / \mathrm{DMF} / \mathrm{H}_{2} \mathrm{O}$ at $20^{\circ} \mathrm{C}$.

point curves obtained for several quasi-binary sections (this means different ratios of dimethylformamide-water as solvent) are given in Figure 3. In Figure 4 , these data have been plotted in a ternary diagram for one temperature $\left(25^{\circ} \mathrm{C}\right)$. 


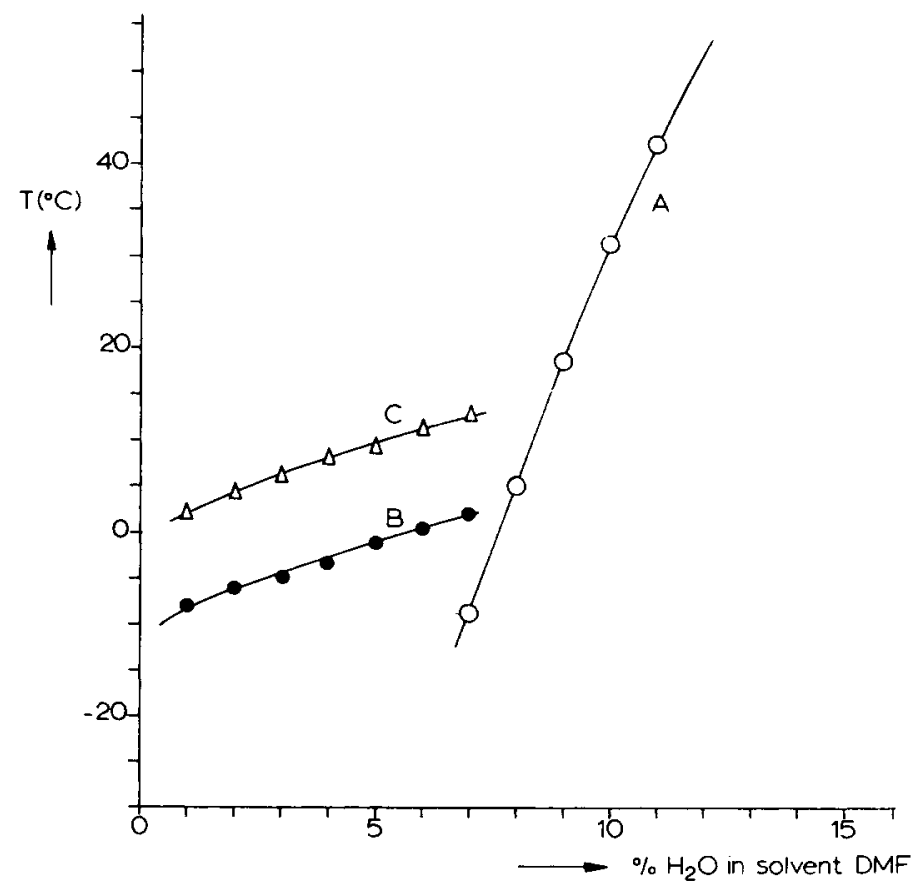

Fig. 5. Phase behavior at low water content in the system $\mathrm{PU} / \mathrm{DMF} / \mathrm{H}_{2} \mathrm{O}$ for a polymer concentration of $25 \%$.

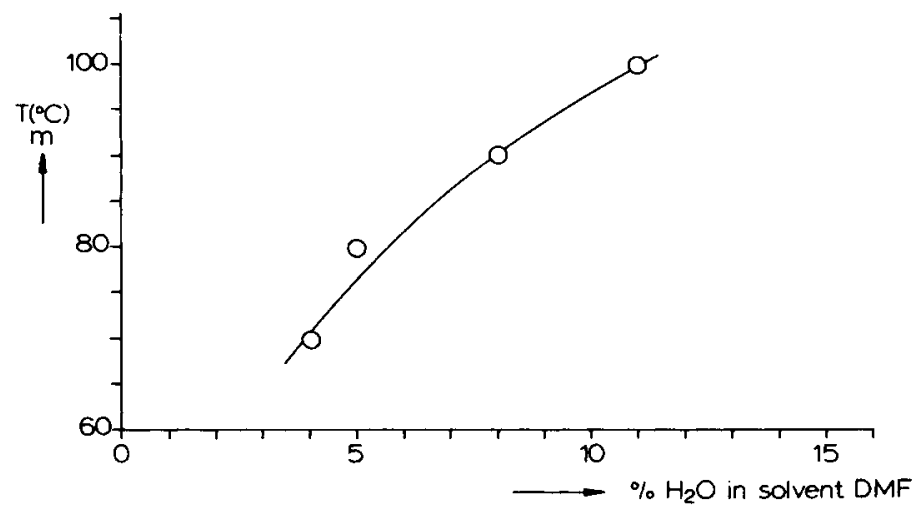

Fig. 6. Gel melting points in the system $\mathrm{PU} / \mathrm{DMF} / \mathrm{H}_{2} \mathrm{O}$ for a polymer concentration of $50 \%$.

If plotted against the increasing concentration of water at one polymer concentration (25\%), curve $A$ in Figure 5 is obtained.

\section{Crystallization}

If there is less than $8 \%$ water in the solvent, the PU solution becomes turbid at a temperature which is dependent on the cooling rate. In Figure 5, curve B corresponds to a very low cooling rate $\left(1^{\circ} \mathrm{C}\right.$ per $\left.24 \mathrm{hr}\right)$. At faster cooling rates, this curve is shifted to lower temperatures.

The phase-separated solution remains fluid. On heating the phase-separated solution, the turbidity disappears again at a temperature corresponding to curve 


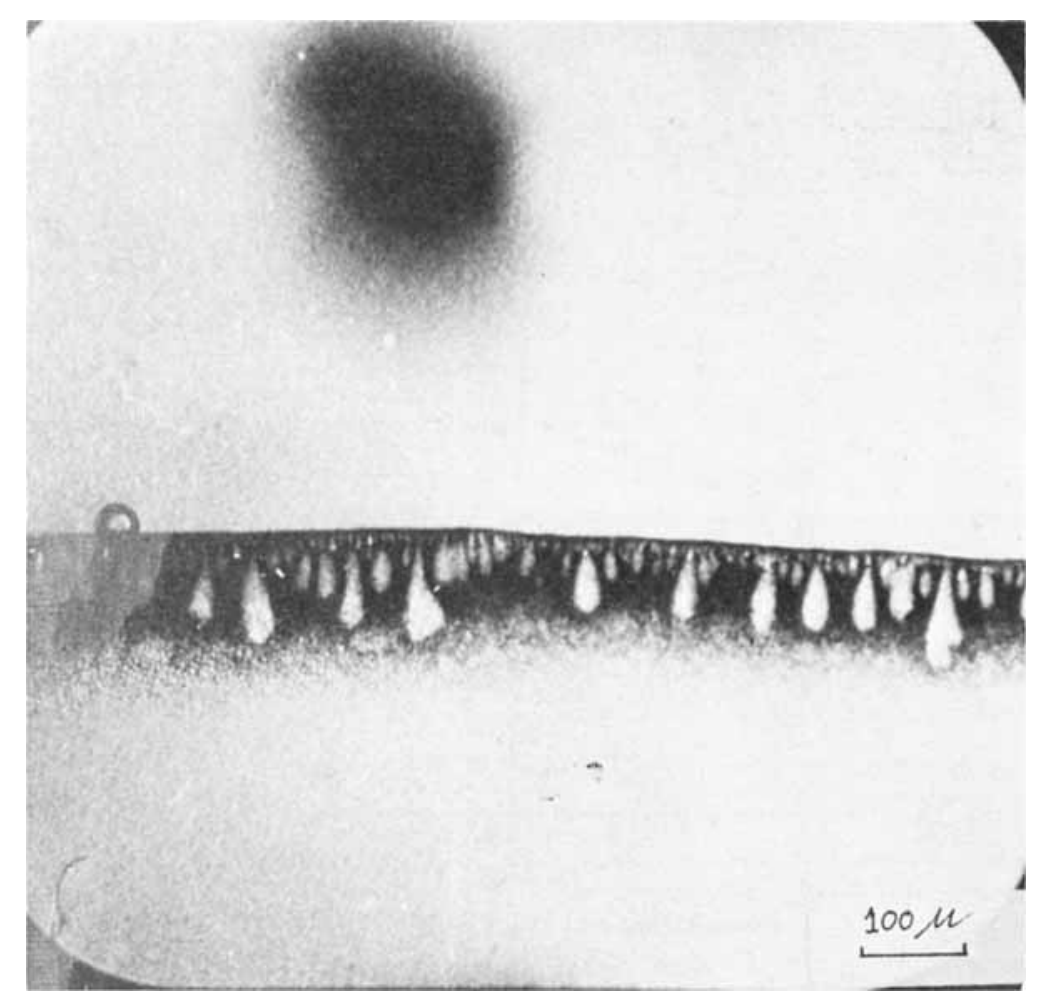

Fig. 7. Coagulation front $8 \mathrm{sec}$ after contact with water (PU concentration $25 \%$ ), as observed with an optical microscope.

C. This gap between cooling and heating curve clearly indicates crystallization, in which undercooling is quite common. ${ }^{16}$ At high polymer concentration, gelation occurs; the kinetics of gelation depend on water content and temperature. The gel is elastic and opaque. Upon heating, the gel becomes fluid at a certain temperature (the gel melting point). In differential scanning calorimetry (DSC), an endothermic peak is obtained on heating. In Figure 6, the gel melting points determined by DSC measurements are given for gels with a polymer content of $50 \%$.

\section{Morphology and Kinetics}

By means of an optical microscope, the coagulation process was studied. Figure 7 was taken $8 \mathrm{sec}$ after water was added. A higher magnification of the coagulation front is given in Figure 8. In Figures 9, 10, and 11, which were taken at time intervals of $1 \mathrm{sec}$, coalescence of droplets can be seen to occur.

In Figure 12, a scanning electron miscroscope photography of a membrane obtained from the system PU in DMF, coagulated in water, is shown. Figure 13 shows a SEM micrograph of a demixed solution in which the polymer concentration was very low $(0.5 \% \mathrm{PU})$. It is obvious that the structure differs from that in Figure 12. In Figure 14, a scanning electron microscope photograph of a membrane obtained from the system modified SIS/1,2-dichlorobenzene/ water-methanol is shown. 


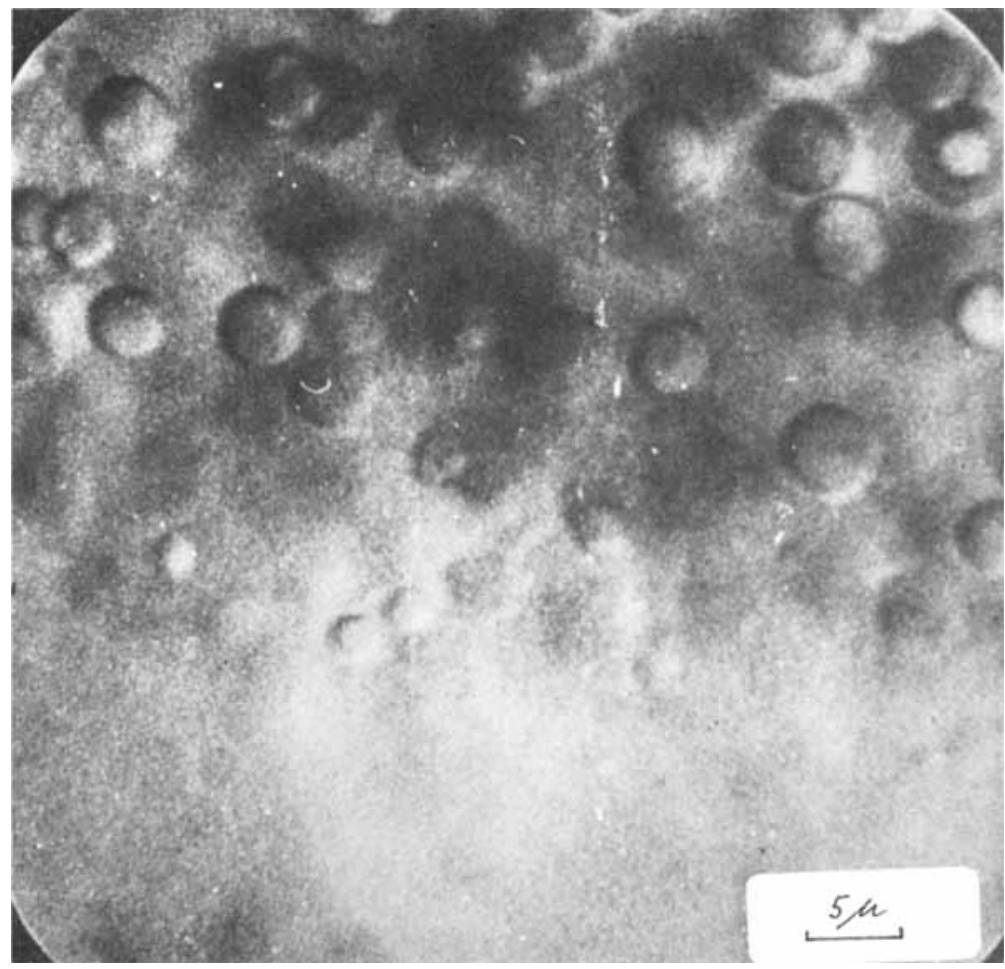

Fig. 8. High magnification of the coagulation front shown in Fig. 7.

As can be seen from Figure 15, the penetration distance of the coagulation front (the liquid-liquid demixing front, not that of the finger-like cavities) is proportional to $\sqrt{t}$. The penetration rate of the coagulation is of the same magnitude here as in the system cellulose acetate-dimethylformamide-water. ${ }^{8}$

\section{Hyperfiltration Experiments}

Hyperfiltration experiments were carried out as described. Typical results of the polyurethane membranes were a salt rejection of $21 \%$ and a flux of 1.1 $\mathrm{cm} / \mathrm{hr}$. The modified SIS membranes showed a salt retention of $62 \%$ and a flux of $2.4 \mathrm{~cm} / \mathrm{hr}$. The membrane performances have not been optimized as yet.

\section{DISCUSSION}

The combination of the optical microscope photographs (Figs. 7-11) and the SEM photographs (Figs. 12 and 14) shows that what is visible as a droplet during the demixing under the microscope is in the final membrane a spherical void in the sponge-like area. The photographs of the coalescent droplets also show that at that particular stage of the coagulation, both phases can still be fluid. The SEM photograph of the product of the coagulation process at very low $(0.5 \%)$ polymer concentration (Fig. 13) shows spherical polymer particles. Following the theoretical section on liquid-liquid phase separation, nucleation and growth of the diluted phase occurs in the first case (Fig. 12). 


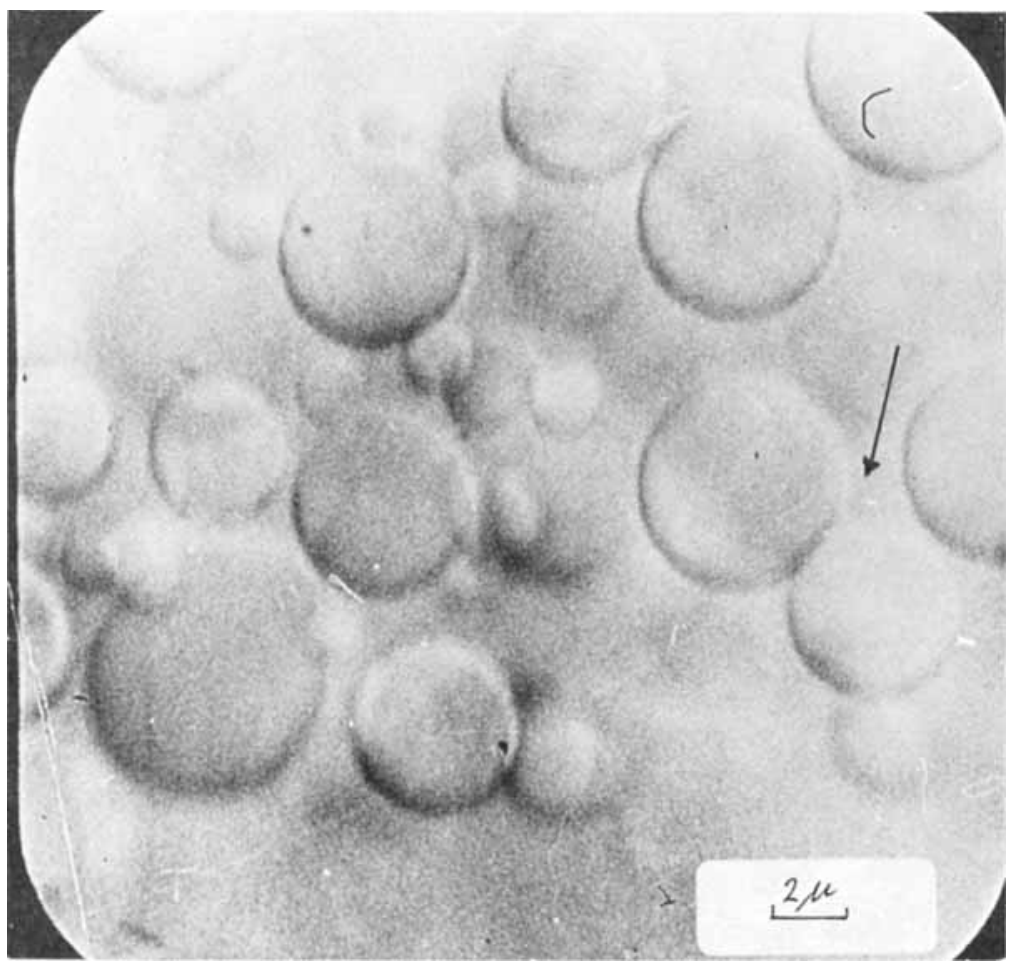

Fig. 9. Coalescence of droplets, optical microscope.

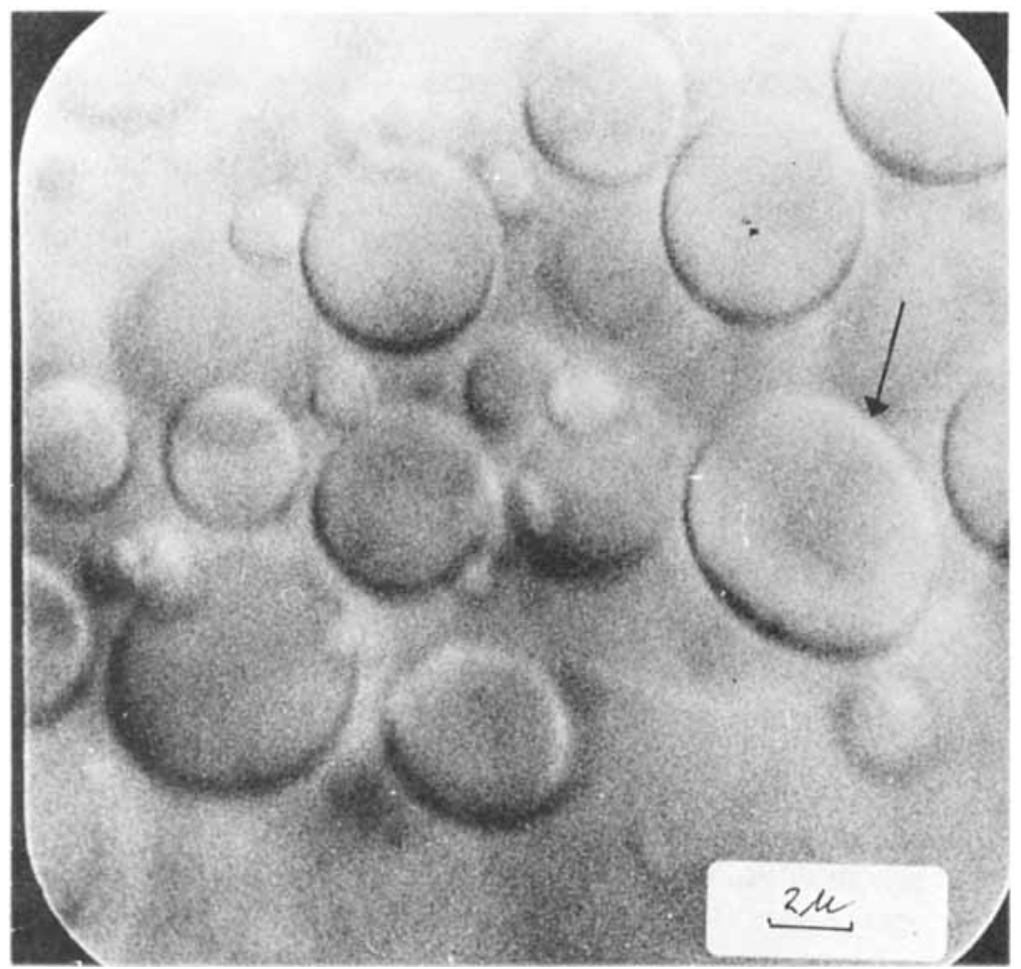

Fig. 10. Coalescence of droplets. Picture taken 1 sec after Fig. 9. 


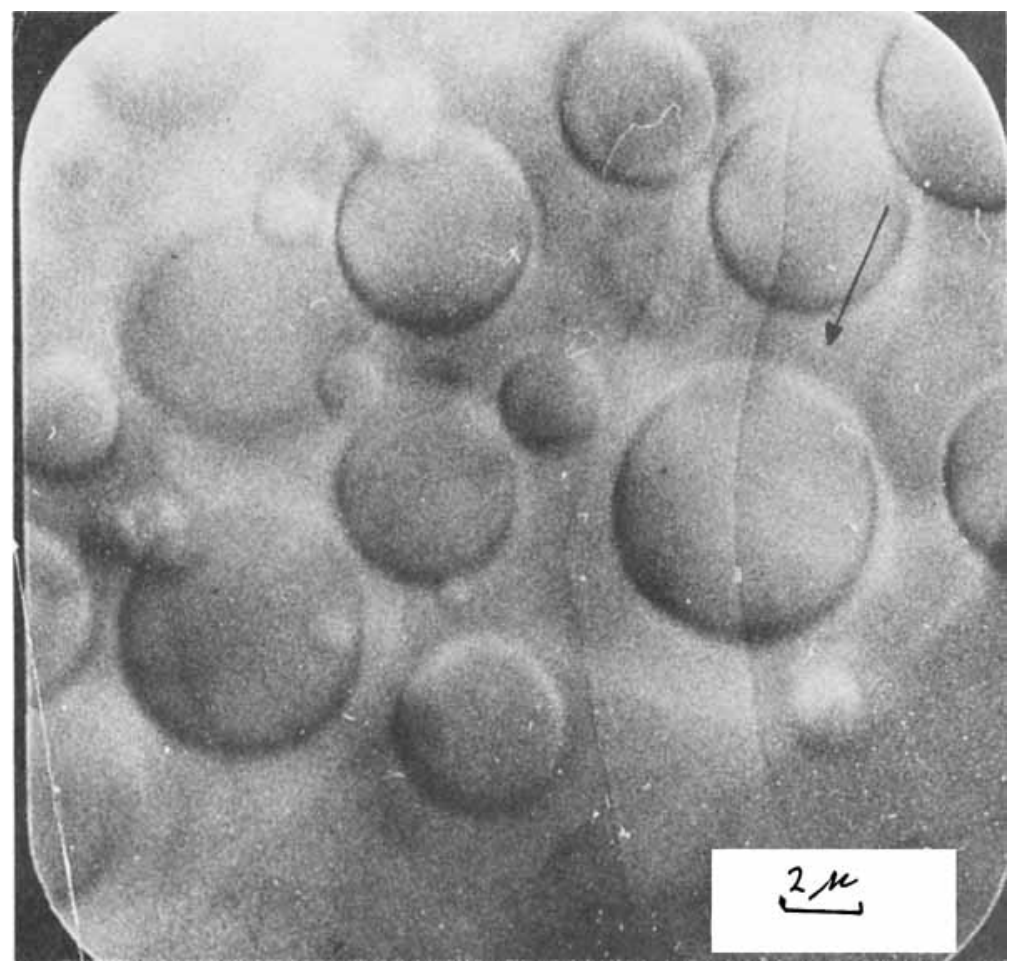

Fig. 11. Coalescence of droplets. Picture taken $1 \mathrm{sec}$ after Fig. 10.

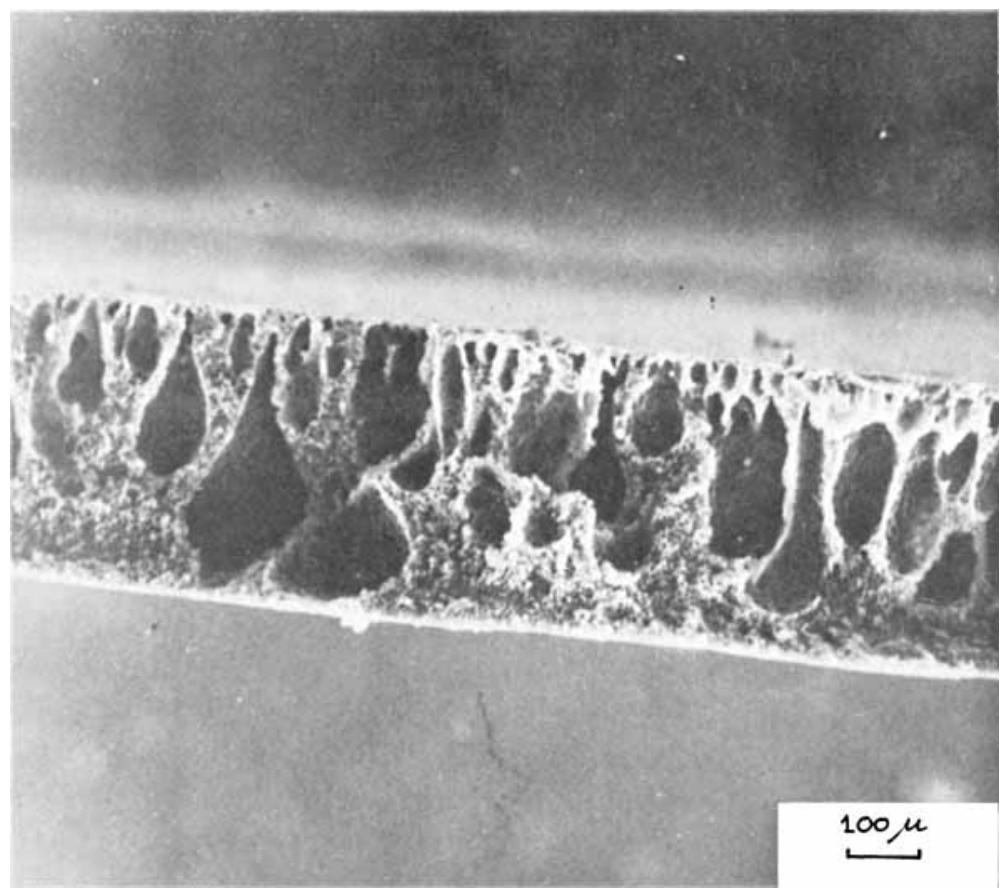

Fig. 12. Hyperfiltration membrane obtained from the system $\mathrm{PU} / \mathrm{DMF} / \mathrm{H}_{2} \mathrm{O}$ (SEM photograph). 


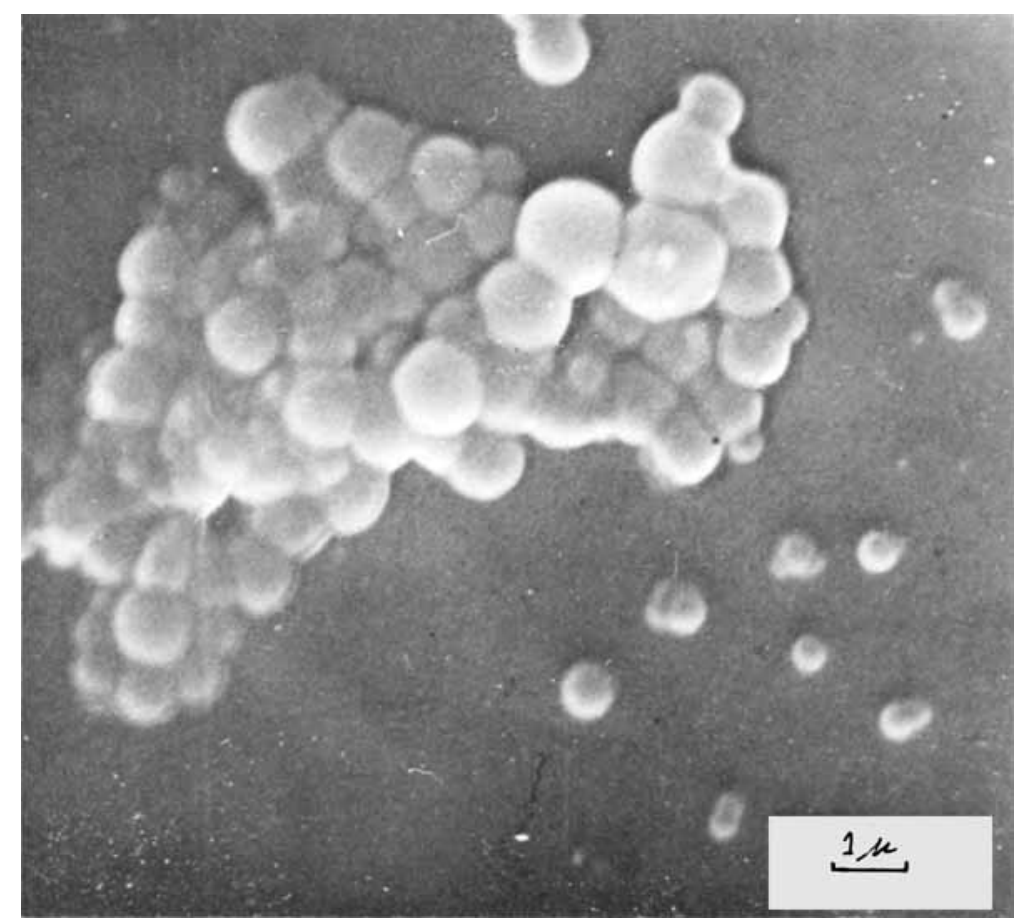

Fig. 13. SEM photograph of the latex particles which occur after the coagulation of a very diluted PU solution.

In the latter case (Fig. 13), nucleation and growth of the concentrated phase has occurred. Nucleation and growth of the diluted phase is observed for the PU system as long as the polymer concentration is above $5 \%$. Therefore, the critical point in Figure 4 lies at a rather low polymer concentration $(<5 \%$ PU).

The SEM photograph of the SIS membrane shows the same sponge-like structure which originates from nucleation and growth of the diluted phase. The structure observed for our membranes has also been found in other types of membranes. ${ }^{7,8,24}$ The explanation of the presence of the finger-like cavities given by Gröbe et al. ${ }^{11}$ seems the most probable for this type of systems. This opinion is mainly based on the observation made by several authors that these fingers can penetrate the solution further than the coagulation front, without causing direct coagulation in the areas between these fingers. $8,11,25,26$

This observation excludes, as the usual mechanism, the cracking of the surface skin layer or the disturbance of this layer and occlusion of nonsolvent. Moreover, the explanation of Gröbe et al. is in agreement with the results of Frommer et al. ${ }^{8}$ who found a bigger chance to obtain finger-like cavities when the nonsolvent and the solvent show a high affinity (big exothermic heat of mixing). This means a large driving force for diffusion of the solvent into these fingers. Frommer also found that the fingers could only grow in the liquid polymer solution. The number of finger-like cavities should be diminished when the difference in chemical potential of the solvent in the polymer solution and the nonsolvent is 


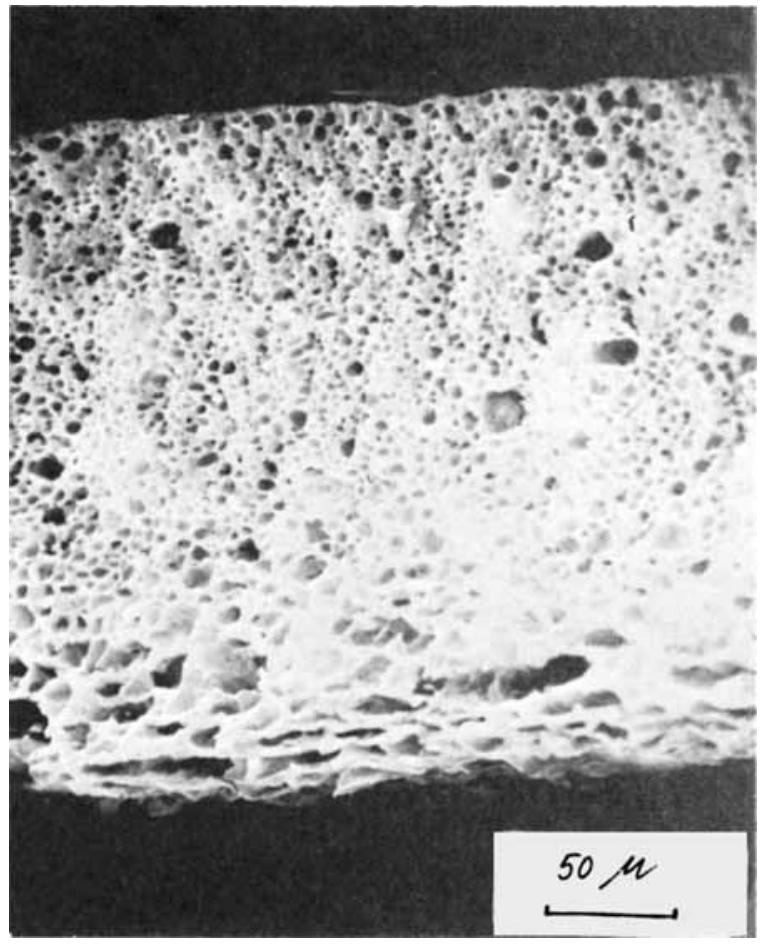

Fig. 14. SEM photograph of a membrane obtained from the system modified SIS/1,2-dichlorobenzene/(water-methanol).

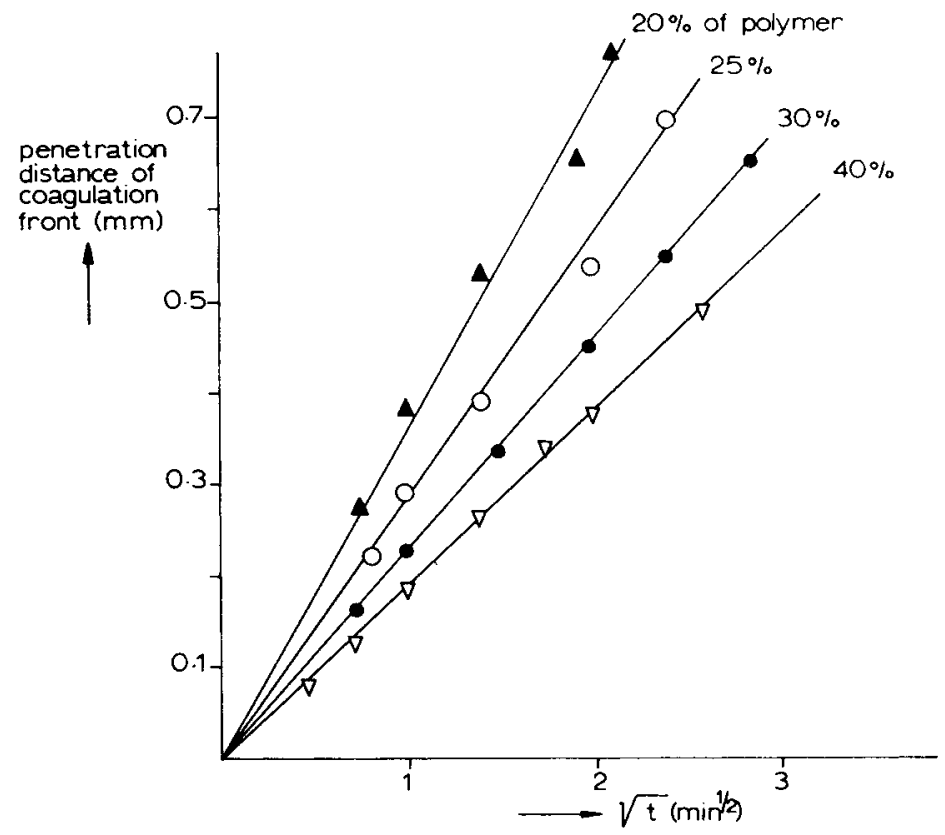

Fig. 15. Penetration of the coagulation front in the $\mathrm{PU} / \mathrm{DMF} / \mathrm{H}_{2} \mathrm{O}$ system. 
lowered. Frommer achieved this by salt-gelling, while we found that in the polyurethane system, adding solvent (DMF) to the nonsolvent (water) was effective in this respect. This effect was also found by Strathmann. ${ }^{7}$

In the SIS system, the solvent (1,2-dichlorobenzene) is not miscible with water, and miscibility is achieved only after adding methanol to the nonsolvent solution. Thus, the affinity between solvent and nonsolvent is low, and a membrane without finger-like cavities is the result (Fig. 14). Of course, the above reasoning can only be approximate because the affinities between solvent and polymer and nonsolvent and polymer have been left out of consideration.

The difficulty in the explanation given by $\mathrm{Matz}^{9}$ is that the surface tensions (valid for the interface between the substance and air) do not give direct information about the interfacial energy between the polymer solution and coagulation bath. Furthermore, it should be argued that the interface becomes less sensitive when the viscosity increases through the loss of solvent to the coagulation bath. At any rate, the explanation is not consistent with the experimental data which show that adding solvent to the coagulation bath gives fewer fingerlike cavities.

The penetration of the coagulation front inward is proportional to $\sqrt{t}$. This is in agreement with all the diffusion equations derived for such situations. ${ }^{7,25,27,28,29}$ When the liquid-liquid phase separation has proceeded through the formation of spherical droplets or finger-like cavities, the polymer matrix will gradually become more concentrated until it eventually solidifies. We feel that the tendency of a polymer solution to crystallize and/or form a gel when high enough in concentration could be very important for the final membrane performance. Indeed, it has been found in cellulose acetate membranes that the ordering in the skin is an important variable. ${ }^{6,30}$

A different type of mechanism might be operative typically for skin formation. Since, in general, the top layer of the film has a higher concentration by the evaporation of solvent and because the rate of phase separation is very high for this layer, in direct contact with nonsolvent, the mechanism of spinodal demixing (see theoretical section) cannot be excluded. The result is a favorable fine-mazed polymer network, intertwined by the fine pores. Crystallization in polymer regions may also follow here.

\section{CONCLUSIONS}

The general mechanism for the membrane formation by coagulation in nonsolvents follows the following scheme:

a. Direct desolvation of a top layer (skin) where most likely gelation takes place (especially when an evaporation step is performed before coagulation).

b. Liquid-liquid phase separation in the solution below the skin. In most instances, nucleation and growth of the diluted phase occurs. In this case the polymer forms the continuous matrix, and the final structure after complete desolvation is the typical sponge-like structure. When the difference in chemical potential for the solvent between the polymer solution and the nonsolvent is large, finger-like cavities also occur.

The authors are grateful to Mr. A. Bakker for carrying out a part of the experiments described in this paper. 


\section{References}

1. S. Loeb and S. Sourirajan, Advan. Chem. Ser., 38, 117 (1962).

2. M. G. Bungenberg de Jong, Kolloid-Z., 80, 221 (1937).

3. K. H. Maier and E. A. Scheuermann, Kolloid-Z., 171, 122 (1960).

4. B. Keilin, Office of Saline Water, Res. Dev. Rept. 117, Aug. 1964.

5. R. E. Kesting, Synthetic Polymeric Membranes, McGraw-Hill, New York, 1971.

6. H. Strathmann, P. Scheible, and R. W. Baker, J. Appl. Polym. Sci., 15, 811 (1971).

7. H. Strathmann, K. Kock, P. Amar, and R. W. Baker, Desalination, 16, 179 (1975).

8. M. A. Frommer and D. Lancet, in Reverse Osmosis Membrane Research, H. K. Lonsdale and

H. E. Podall, Eds., Plenum Press, New York, 1972.

9. R. Matz, Desalination, 10, 1 (1972).

10. J. P. Craig, J. P. Knudsen, and V. F. Holland, Text. Res. J., 32, 435 (1962).

11. V. Gröbe, G. Mann, and G. Duwe, Faserforsch. Textiltech., 17, 142 (1966).

12. D. M. Koenhen, M. A. de Jongh, C. A. Smolders, and N. Yücesoy, Colloid Polym. Sci., 253, $521(1975)$.

13. P. J. Flory, Principles of Polymer Chemistry, Cornell University Press, Ithaca, N.Y., 1953.

14. J. W. Cahn, Trans, Met. Soc. AIME, 242, 166 (1968).

15. C. A. Smolders, J. J. van Aartsen, and A. Steenbergen, Kolloid-Z.Z. Polym., 243, 14 (1971).

16. L. Mandelkern, Crystallization of Polymers, McGraw-Hill, New York, 1964.

17. P. H. Morgan and G. S. Park, Faraday Disc. Chem. Soc., 57, 38 (1975).

18. D. Eagland, P. Dilling, and R. G. Wheeler, Faraday Disc. Chem. Soc., 57, 181 (1975).

19. T. A. Bryce, A. A. McKinnon, E. R. Morris, D. A. Rees, and D. Thorn, Faraday Disc. Chem. Soc., 57, 221 (1975).

20. D. S. Reid, T. A. Bryce, A. H. Clark, and D. A. Rees, Faraday Disc. Chem. Soc., 57, 230 (1975).

21. V. M. Averyanova and G. N. Tunofeyeva, 12th IUPAC Microsymposium on Macromolecules cont. F4, 1973.

22. P. T. van Emmerik and C. A. Smolders, J. Polym. Sci. C, 38, 73 (1972).

23. P. M. van der Velden, M. H. V. Mulder, L. van der Does, and C. A. Smolders, Polym. Letters, 14, 5 (1976).

24. G. J. Gittens, P. A. Hitchcock, and G. E. Wakley, Desalination, 12, 813 (1966).

25. M. E. Epstein and A. J. Rosenthal, Text. Res. J., 36, 813 (1966).

26. M. van Hilten, T. H. Twente, Report 1973.

27. D. R. Paul, J. Appl. Polym. Sci., 12, 383 (1968).

28. H. Jost, A. Gröbe, and H. Klare, Faserforsch. Textiltech., 14, 522 (1963).

29. A. Rende, J. Appl. Polym. Sci., 16, 585 (1972).

30. R. Matz, Desalination, 12, 273 (1973).

Received December 15, 1975 